Test Method

\title{
Evaluation of in-situ shrinkage and expansion properties of polymer composite materials for adhesive anchor systems by a novel approach based on digital image correlation
}

\author{
Gerald Singer $^{\mathrm{a}, \mathrm{b}}$, Gerhard Sinn ${ }^{\mathrm{b}}$, Helga C. Lichtenegger ${ }^{\mathrm{b}}$, Stefan Veigel $^{\mathrm{c}}$, Michele Zecchini ${ }^{\mathrm{a}}$, \\ Roman Wan-Wendner ${ }^{\mathrm{a}, \mathrm{d}, *}$ \\ ${ }^{a}$ University of Natural Resources and Life Sciences Vienna, Christian Doppler Laboratory LiCRoFast, Institute of Structural Engineering, Peter-Jordan-Straße 82, 1190, \\ Vienna, Austria \\ ${ }^{\mathrm{b}}$ University of Natural Resources and Life Sciences Vienna, Institute of Physics and Materials Science, Peter-Jordan-Straße 82, 1190, Vienna, Austria \\ ${ }^{\mathrm{c}}$ University of Natural Resources and Life Sciences Vienna, Institute of Wood Technology and Renewable Materials, Konrad Lorenz-Straße 24, 3430, Tulln an der Donau, \\ Austria \\ ${ }^{\mathrm{d}}$ Department of Structural Engineering, University Ghent, Belgium
}

\section{A R T I C L E I N F O}

\section{Keywords:}

Chemical shrinkage

Thermal expansion

Digital image correlation (DIC)

Polymer-matrix composites (PMC)

Adhesive anchor

\begin{abstract}
A B S T R A C T
The curing reaction of thermosetting resins is associated with chemical shrinkage which is overlapped with thermal expansion as a result of the exothermal enthalpy. Final material properties of the polymer are determined by this critical process. For adhesive anchor systems the overall shrinkage behavior of the material is very important for the ultimate bond behavior between adhesive and the borehole wall. An approach for the insitu measurement of 3-dimensional shrinkage and thermal expansion with digital image correlation (DIC) is presented, overcoming the common limitation of DIC to solids. Two polymer-based anchor systems (filled epoxy, vinylester) were investigated and models were developed, showing good agreement with experimental results. Additionally, measurements with differential scanning calorimetry (DSC) provided supporting information about the curing reaction. The vinylester system showed higher shrinkage but much faster reaction compared to the investigated epoxy.
\end{abstract}

\section{Introduction}

Adhesive anchor systems are based on polymer matrix systems in combination with different filler materials and additives in order to modify their properties [1] in terms of mechanical stability during their service life [2,3], curing and aging behavior [4-6] and compatibility with targeted materials in the application. Chemical shrinkage during the curing reaction is a well-known phenomenon of thermosets $[7,8]$ but it is usually overlapped with thermal expansion [9] since the crosslinking is an exothermal process. Both contributions, chemical shrinkage and thermal expansion, determine the residual strains and stresses and the final dimensions of the cured material [10]. In fact, it is hard to distinguish between these simultaneous processes since they influence each other. The performance and life-time of installed adhesive anchors ultimately depends on the residual strain state that is reached at the end of the curing process. The reason are tensile eigenstresses that will develop in a confined situation such as a borehole if any final shrinkage or expansion is observed. In case of shrinkage these correspond macroscopically to tensile stresses on the interface between steel anchor element and borehole and will limit the achievable macroscopic shear bond strength.

The curing process of thermosets can be divided into three characteristic regions [11]. First the mobile monomer molecules exist in a liquid and behave like a viscous fluid. Their unit volume is defined by molecule size, Van-der-Waals volume and Brownian motion at the given temperature [12]. In the second region, the monomers form chains and mainly grow in linear branches, which then form cross-links in the third and final region, leading to a 3-dimensional rigid network. This effect is connected to a formation of covalent bonds, reducing the specific volume of the system. The extent of shrinkage can be assumed to be directly proportional to the degree of cure within a specific formulation. Depending on the monomers, hardener and chemistry of the

\footnotetext{
* Corresponding author. University of Natural Resources and Life Sciences Vienna, Christian Doppler Laboratory LiCRoFast, Institute of Structural Engineering, Peter-Jordan-Straße 82, 1190, Vienna, Austria.

E-mail address: roman.wanwendner@ugent.be (R. Wan-Wendner).
} 
curing reaction (radical polymerization, poly-addition, poly-condensation), different amounts of shrinkage will be obtained due to covalent bond formation and side products. The type of chemical reaction also has a direct influence on the polymerization rate (conversion rate), as does the stoichiometric ratio of reactants.

Thermal expansion during the curing reaction of a thermoset can be simply explained by the molecular movement due to thermal energy. The higher the degree of cure, the lower the space of free motion becomes. Highly exothermic reactions lead to a significant contribution of thermal expansion throughout the curing process and may cause residual stresses in the material after cooling down, involving stress relaxation processes [13]. Molecular modeling approaches can be used to predict the estimated curing shrinkage under specific conditions. For instance group interaction modeling (GIM) was shown to predict acrylate and methacrylate polymerization shrinkage in agreement with experimental data [14]. However, local deviation from the conditions, chosen for the calculation should be considered for practical application.

\subsection{Measurement of cure shrinkage of thermosets}

Several techniques have been described for measuring cure shrinkage of thermosetting resins. They can be categorized into two general approaches: linear shrinkage measurement and volume dilatometry [15]. Measuring dimensional changes during the curing reaction in only one dimension (axial) requires making assumptions in order to derive volumetric shrinkage. It was shown that for different axial measurement methods ("bonded disc method" and "non-bonded" free shrinkage method) the sample geometry, adhesion properties and anisotropy of the shrinkage are important factors when calculating the volumetric shrinkage [16]. Considering these effects, different results may be obtained from linear shrinkage measurement, depending on the chosen method. In contrast, a volume dilatometer gives direct 3-dimensional information about volume change. However, toxic mercury or water that could potentially influence the shrinkage was commonly used in former instruments and pycnometers that are filled with inert gas (e.g. He) became more important [17]. It has to be mentioned that such pycnometers only determine the final shrinkage under certain ambient conditions. Hence, this method cannot be used for in-situ shrinkage monitoring.

Further approaches describe shrinkage measurement using a rheometer [8], a method based on changes in time of travel of longitudinal ultrasonic waves enables the determination of shrinkage and modulus of thermosetting resins [18] or an optical method using contour videoimaging of a drop of resin [19]. These approaches mentioned represent interesting developments for thermosetting shrinkage measurement. However, they are characterized by certain advantages and disadvantages, depending on the case of application. For instance, they could not be applied to determine the shrinkage of the resin in a chemical anchor system that is installed in a borehole.

\subsection{Measurement of thermal expansion of thermosets}

The linear coefficient of thermal expansion (CTE) can be quantified by measuring the dimensional change in length of a material in dependence on the temperature. Possible phase changes at a certain temperature, such as the glass transition point $\left(T_{g}\right)$ should be considered for the definition of the temperature range for the measurement.

A well-established method to measure thermal expansion is the mechanical dilatometer, which is based on a mechanical sensor that monitors the displacement while the sample is heated in a surrounding furnace. This technique is relatively simple but requires precise instruments and direct contact to the specimen. Contactless thermal expansion can be measured via optical methods, such as optical interference that is based on calculating the path difference between laser beams when the sample expands. Another possibility is represented by speckle pattern interferometry using an interference pattern that is created on the specimen's surface by two collimated laser beams. The changes of the pattern are recorded by a camera when the surface expands due to changes in temperature [20]. This techniques can be used up to relatively high temperatures of up to $3300{ }^{\circ} \mathrm{C}$ using a special setup developed by Miiller and Cezairliyan [21]. However, expensive equipment for the laser generation and a Michelson interferometer is necessary.

\subsection{Principal of digital image correlation (DIC)}

A digital image correlation (DIC) system provides contactless optical evaluation of strain and displacement of a material. For 3D-measurements, two cameras record the movement of an irregular pattern on the surface of the specimen from different angles. Processing the recorded images by means of software provides information about the surface displacement and allows the construction of full field strain maps in 3 dimensions [22]. The resolution mainly depends on the quality of the speckle pattern on the surface of the specimen, light conditions and distance as well as resolution of the cameras. DIC can be used for outdoor applications on large scale such as civil engineering structures as well as for laboratory investigations of small samples. A wide range and scale of applications as well as relatively low costs for the systems are advantages of the DIC technique. DIC finds widespread application but is mostly limited to solids that allow the application of high-contrast speckle patterns. The literature on strain measurements during chemical curing reactions is scarce in comparison. A one-camera DIC system was already used to measure chemical and thermal shrinkage of a resin after gelation [23]. However, the 3D information was not included in this data and the resin was pre-heated before starting the measurement. In this investigation, a simple yet robust method for the application of a speckle pattern on the surface of still liquid adhesives was successfully applied and can be recommended.

In this study, the curing reaction of two different adhesive anchor systems with high content of inorganic fillers are investigated by DIC and a model was developed considering both effects, the chemical shrinkage due to the crosslinking reaction and thermal expansion, caused by the exothermal process. One adhesive system reacts within several minutes and the second system cures on a timescale of hours. We present a two-camera DIC system as powerful method for measuring 3-dimensional changes in dimensions as an in-situ and contactless approach.

For practical applications this method provides a powerful tool to check the actual shrinkage of the adhesive under certain environmental conditions for the installation of anchor systems in construction work in order to improve quality and safety standards. The formation of the interfaces between concrete borehole, adhesive and steel anchor is an important factor that influences the final capacity of the anchor system, which is dependent on the shrinkage/expansion behavior of the polymer among others and has to be taken into account since conditions in real applications may vary significantly.

\section{Material and methods}

\subsection{Materials}

Two industrially produced polymer-based adhesive anchor systems were tested. One with vinylester and the other with epoxy matrix, both containing relatively high amounts (20 - $50 \mathrm{vol} \%$ ) of inorganic filler materials (quartz/cement) with different grain size between 5 and $300 \mu \mathrm{m}$. The vinylester system consists of methacrylate monomers and dibenzoyl peroxide (DBPO) hardener, the epoxy system is based on bisphenol-A/F and $m$-xylylenediamine hardener.

\subsubsection{Sample preparation}

The materials were obtained in cartridges with two separated 

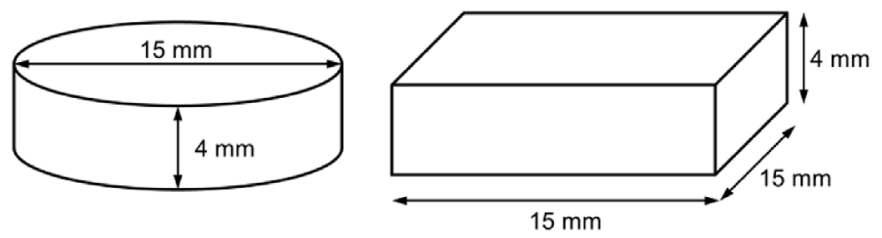

Fig. 1. Sample geometry in round and squared shape.

\section{Principle of setup for shrinkage measurement with DIC}

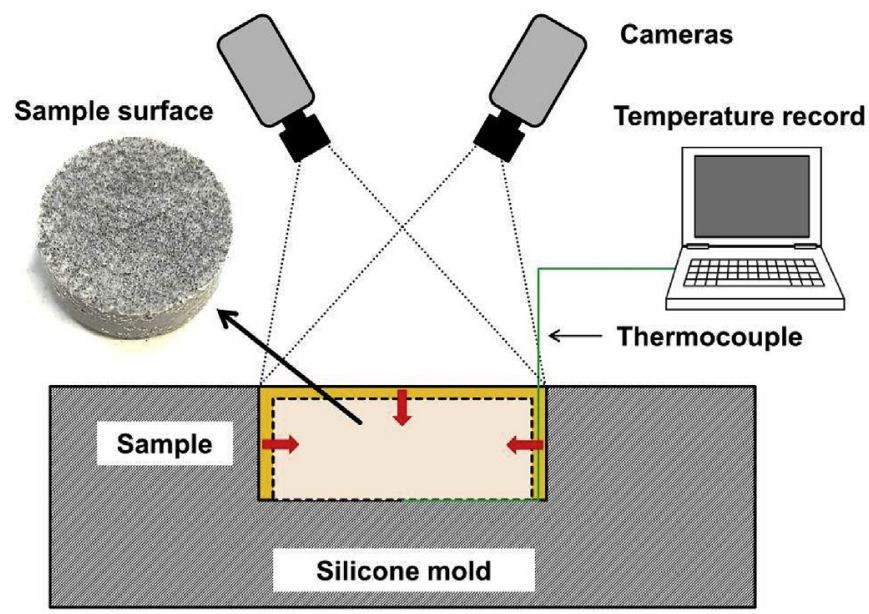

Fig. 2. Schematic illustration of shrinkage measurement using digital image correlation.

reservoirs for resin and hardener. With a press the materials were pushed through a screwed-on mixer tip that automatically ensures the required mixing ratio. The mixture was directly transferred into a silicone mold with round sample geometry of $15 \mathrm{~mm}$ diameter and $4 \mathrm{~mm}$ thickness and squared sample geometry $15 \times 15 \times 4 \mathrm{~mm}^{3}$ as it is shown in Fig. 1. The mold was coated with PTFE spray before in order to avoid adhesion of the material to the silicone. Material excess was removed and fine SiC powder was uniformly sprinkled on the smooth surface of the sample to provide a statistical speckle pattern for the DIC measurement. On the bottom of the silicone mold a thin K-type thermocouple wire was placed and the temperature was recorded during the whole experiment (see Fig. 2).

\subsection{Methods}

\subsubsection{Shrinkage measurement}

A digital image correlation (DIC) system was used to analyze the 3dimensional deformation of the shrinking sample. Two cameras were focused on the sample surface, placed in a Teflon coated silicone mold, recording the whole curing reaction.

\subsubsection{Thermal expansion measurement}

A piece of a cured sample, sprayed with a white paint as undercoating and a fine black speckle pattern for optimal contrast, was placed on a plate of a heater. The temperature was measured with a thermocouple and was used as feedback signal for the heat-controller. DIC images were recorded while the temperature of the sample was increased slowly. To calculate the coefficient of thermal expansion (CTE), the strain from the images of the sample was correlated with the temperature difference between the sensor signal and the environmental value.

\subsubsection{DSC measurements}

Differential scanning calorimetry (DSC) measurements were
Principle of setup for CTE measurement with DIC

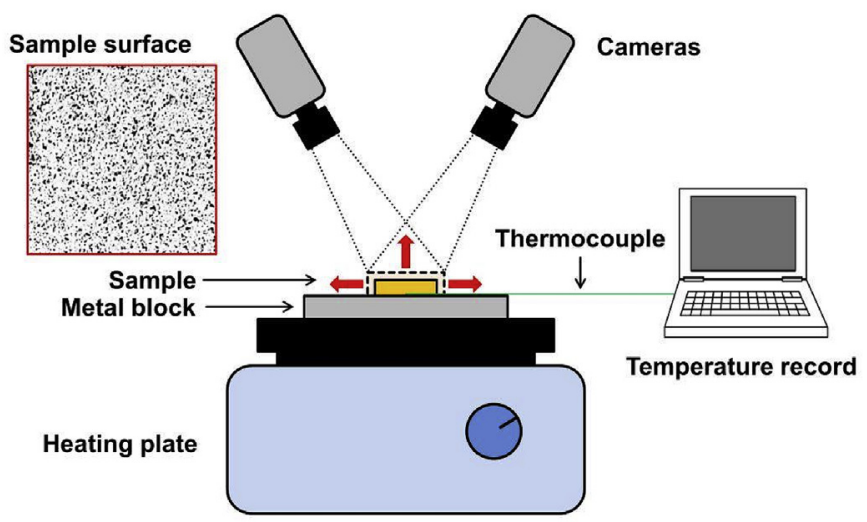

Fig. 3. Schematic illustration of thermal expansion measurement using digital image correlation.

performed on a Netzsch DSC F3 Maia (Netzsch-Gerätebau GmbH, Germany). Samples were measured isothermally at $30^{\circ} \mathrm{C}$ in a closed $40 \mu \mathrm{L} \mathrm{Al}$ crucible. For data evaluation, the software Proteus (NetzschGerätebau GmbH, Germany) was used.

The samples were measured as fresh mix of resin and hardener by pressing both components out of a cartridge using a mixer tip. A small amount of homogenous mix (ca. $10 \mathrm{mg}$ ) were weighed in a crucible, closed with a lid and placed in the DSC. A comparable amount of fully cured sample was used as reference (see Fig. 3).

\section{Results and discussion}

\subsection{Shrinkage behavior}

The DIC shrinkage measurements and the monitored temperature are plotted in Fig. 4. The temperature difference is given by the heat release due to the curing reaction, compared to the environmental temperature. The reason why this temperature difference does not start from zero is the fast reaction of the polymer that already starts when resin and hardener are mixed and hence the temperature of the polymer is already slightly higher than the environmental when the measurement was started. For the epoxy the shrinkage in terms of engineering principal strains on the sample surface showed lower values than the vinylester. In general, the curing reactions of both materials take place on a different time scale with a total curing time of around $300 \mathrm{~min}$ for the epoxy and less than $10 \mathrm{~min}$ for the vinylester. The reaction of the epoxy is characterized by a maximal temperature difference of $7{ }^{\circ} \mathrm{C}$ above room temperature with a relatively high resulting thermal expansion lasting around $120 \mathrm{~min}$, followed by a shrinkage reaction that is connected to a decreasing temperature (Fig. $4 \mathrm{a}, \mathrm{c}$ ). In contrast, the vinylester shows a fast increase of temperature within around $3 \mathrm{~min}$ that has a maximum of $50{ }^{\circ} \mathrm{C}$ above room temperature (Fig. 4b, d). Only a small thermal expansion can be detected within the initial period of fast exothermal reaction and after that, a fast shrinkage reaction takes place for the next 5-7 min.

In Fig. 5 examples of visual representations of strains on the sample surface during the curing process are shown. Some small areas (holes) could not be evaluated because of missing optical information from the speckle pattern. The image in Fig. 5a shows a round epoxy sample after $\sim 26 \mathrm{~min}$ and Fig. $5 \mathrm{~b}$ a squared vinylester sample after $\sim 10 \mathrm{~min}$ from the start of the DIC measurement. The total shrinkage of the epoxy was slightly different for the two geometries, showing $0.6 \mathrm{~mm} / \mathrm{m}(0.06 \%)$ and $0.9 \mathrm{~mm} / \mathrm{m}(0.09 \%)$ for the round and square shape, respectively. This discrepancy could be attributed to the different shape but is expected to be within the accuracy of the measurement for the chosen 
(a) EP, round

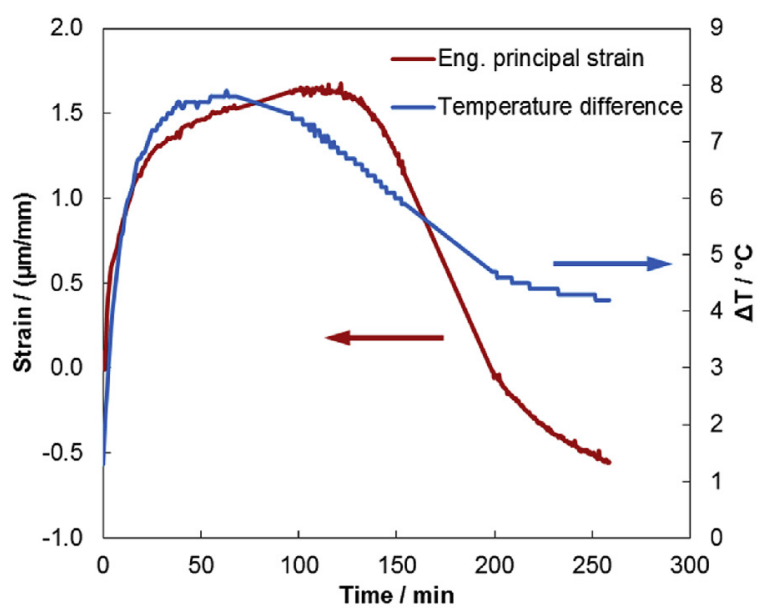

(c) EP, square

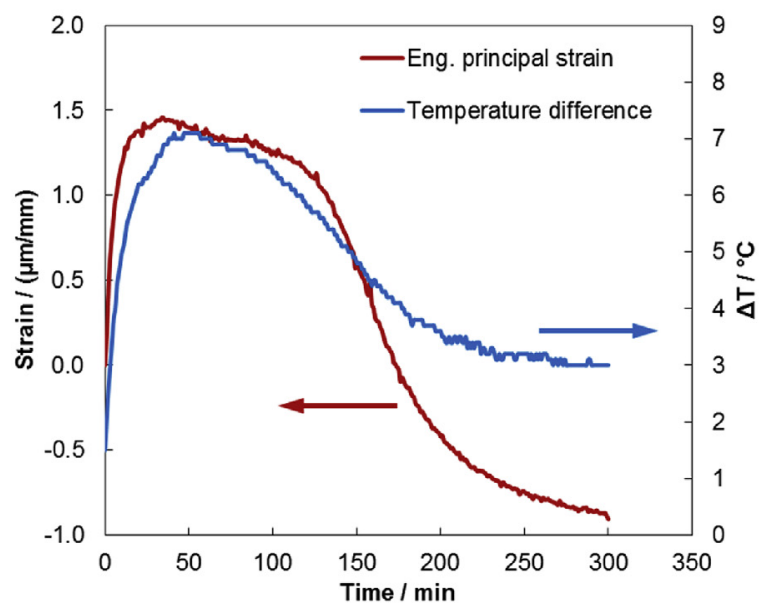

(b) VE, round

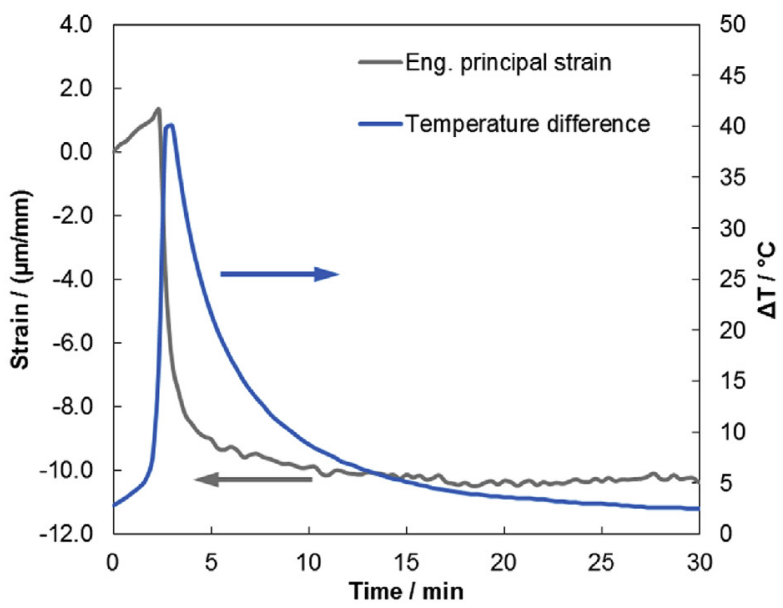

(d) VE, square

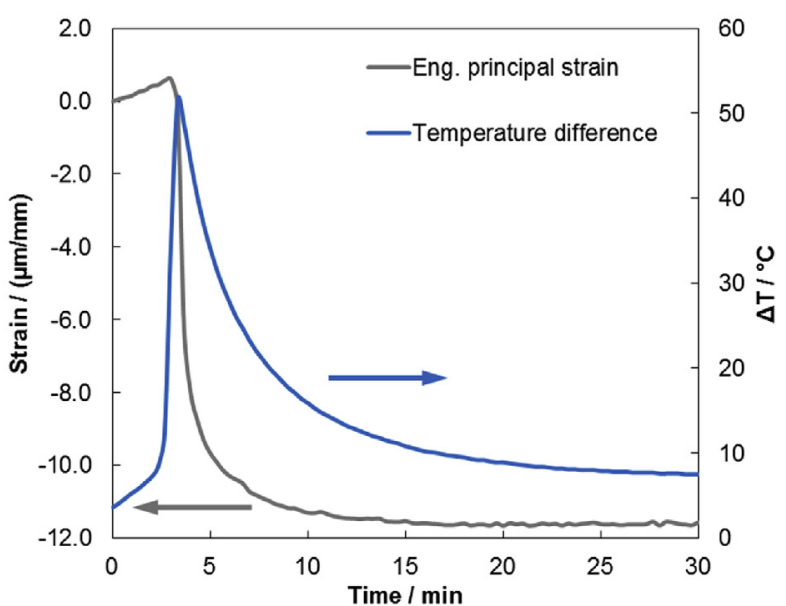

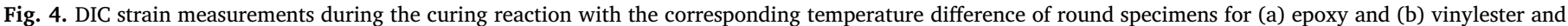
square geometry for (c) epoxy and (d) vinylester.

specimen dimensions. However, for larger samples or structural components shape effects may become significant if there are gradients present in degree of conversion and consequently material properties, temperature, or humidity. Small deviations were also found for the shrinkage of the vinylester for different shapes. For the round geometry $10.5 \mathrm{~mm} / \mathrm{m}(1.05 \%)$ and for the square $11.7 \mathrm{~mm} / \mathrm{m}(1.17 \%)$ were (a) Epoxy: DIC shrinkage (round)

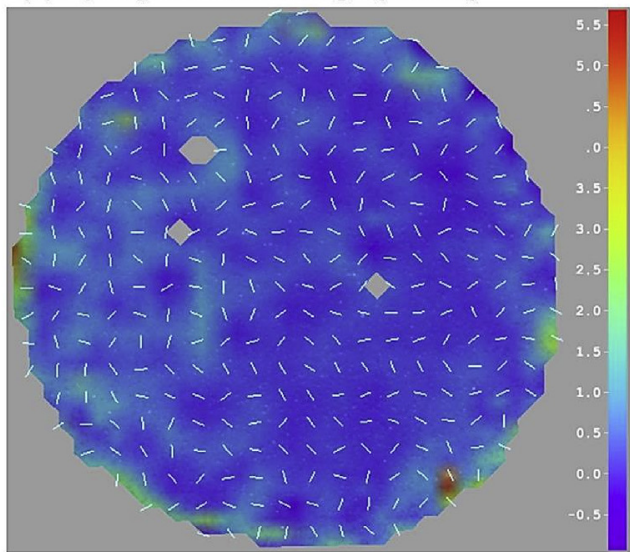

(b) Vinylester: DIC shrinkage (square)

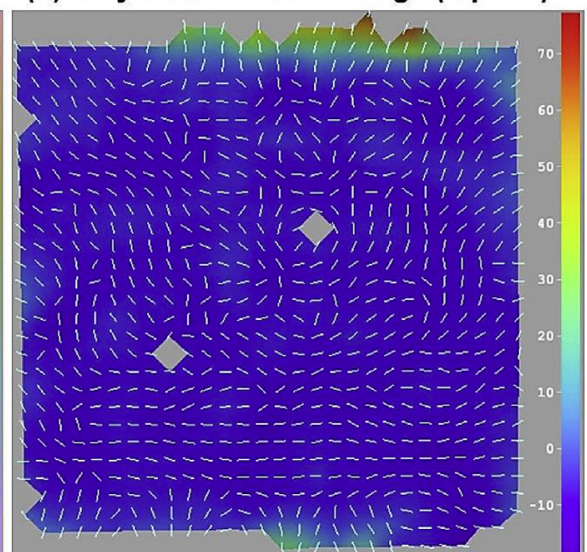

Color scale indicates engineering principal strains, values in Strain/mStrain

Fig. 5. Visualization of the engineering principal strains on the surface obtained from DIC measurements of (a) epoxy sample in round geometry and (b) vinylester sample in squared geometry. The unit Strain/mStrain can be interpreted as shrinkage in $\mu \mathrm{m} / \mathrm{mm}$ or $\mathrm{mm} / \mathrm{m}$. 
(a) DSC ramp test on epoxy

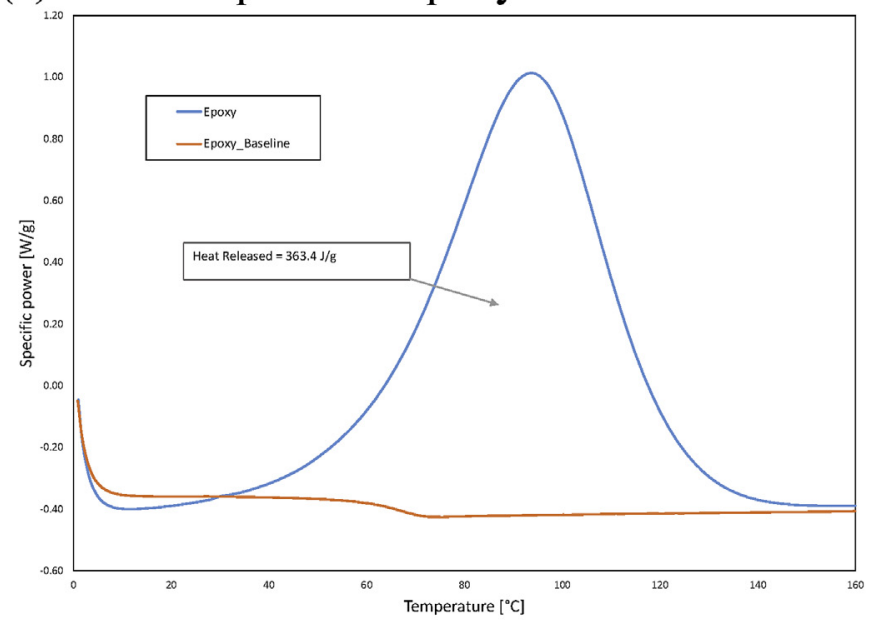

(b) DSC ramp test on vinylester

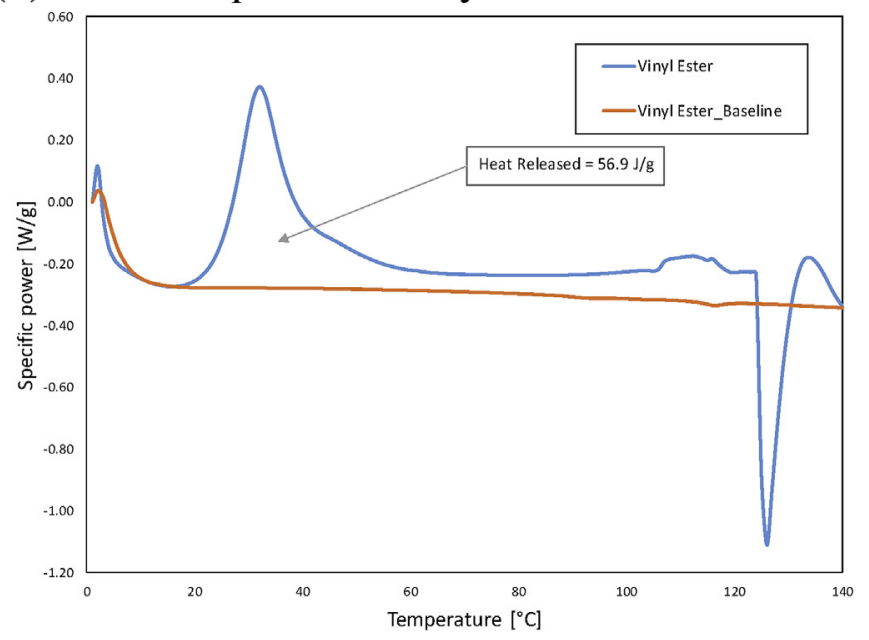

Fig. 6. DSC ramp measurements of the epoxy-based (a) and the vinylester-based (b) material of the fresh mix from the cartridge.

obtained for the total shrinkage. The observed differences due to specimen geometry and inherent measurement uncertainties are significantly smaller than the observed 1 order of magnitude difference between the two materials - epoxy and vinylester. In both cases the engineering principal strains on the surface are distributed homogenously for round and square geometry, except for some small spots on the edges that can be attributed to adhesion effects to the mold. However, these adhesion effects are rather low due to the PTFE spray that was used as release agent.

Results obtained for the shrinkage of both thermosets (epoxy and vinylester) correspond to expectations from literature. For usual unfilled epoxy a total curing shrinkage between $2 \%$ and $6 \%$ was reported [15]. Usually unsaturated polyesters show more curing shrinkage than epoxy, within a range of $7-10 \%$ [24]. When using filler material shrinkage can be significantly reduced [25]. Common filler materials like sand, silica, clay, quartz, alumina or metal powders are added to reduce the shrinkage behavior among others [26].

\subsubsection{DSC measurements}

From the DSC ramp tests performed between $1{ }^{\circ} \mathrm{C}$ and $160{ }^{\circ} \mathrm{C}$ with a heating rate of $10 \mathrm{~K} / \mathrm{min}$ as shown in Fig. 6 , the reaction enthalpy $(\Delta H)$ of the full curing reaction was obtained as difference between the first measurement and the baseline obtained in a second ramp test of the same already cured specimen. The measurements were repeated thrice in order to quantify measurement uncertainties. In case of both materials an exothermal reaction is observed, since chemical energy is released from the material due to the formation of more stable chemical bonds between reactive groups. It can be observed that for the epoxy the curing reaction is much slower and much more exothermal $(\Delta H \approx-361 \pm 1 \% \mathrm{~J} / \mathrm{g})$ than for the vinylester $(\Delta H \approx-55 \pm 4 \% \mathrm{~J} /$ $\mathrm{g}$ ) at the same temperature of $30^{\circ} \mathrm{C}$. The reaction of the epoxy (Fig. 6a) and vinylester (Fig. 6b) finish within approximately $6 \mathrm{~h}$ and $20 \mathrm{~min}$, respectively. This curing behavior can be attributed to the nature of the different chemical reactions. The epoxy curing reaction is based on an addition reaction of amine-groups of the hardener to the epoxy-groups of the resin, whereas the vinylester reacts via a radical mechanism between double-bonds of the side chains.

In terms of shrinkage behavior, the exothermic nature of the curing reaction is important information in order to differentiate between chemical shrinkage and thermal expansion since the optical strain measurements contain both components. Initially, thermal strains are determined by the rapid heat release following the DSC signal. The second phase of the shrinkage measurements is influenced by the cooling behavior after the reaction has finished which is determined by thermal conductivity of the material and natural heat exchange with the environment.

\subsection{Thermal expansion}

The coefficient of thermal expansion (CTE) was obtained by plotting the strain over temperature. The slope of a linear fit represents the CTE at different states of the polymers. In Fig. 7a the CTE of the epoxy between $30^{\circ} \mathrm{C}$ and $57^{\circ} \mathrm{C}$ is in the range of $0.05 \mathrm{~mm} / \mathrm{mK}$ and above that temperature the CTE increases by one order of magnitude to about $0.5 \mathrm{~mm} / \mathrm{mK}$ between $59^{\circ} \mathrm{C}$ and $64^{\circ} \mathrm{C}$. The CTE of the vinylester in Fig. $7 \mathrm{~b}$ changes from around $0.03 \mathrm{~mm} / \mathrm{mK}$ between $26^{\circ} \mathrm{C}$ and $50{ }^{\circ} \mathrm{C}$ to the double value of $0.06 \mathrm{~mm} / \mathrm{mK}$ between $50^{\circ} \mathrm{C}$ and $78^{\circ} \mathrm{C}$. Between the two states of the polymers a certain transition region can be identified. For the epoxy some data points were excluded for the regression, marked with triangles. The correlation coefficient $\left(R^{2}>0.97\right)$ indicates a good fit for both regions. Data of the vinylester measurement showed scattering and the fit quality is lower $\left(R^{2}>0.90\right)$. Probably surface reflections and less optimized light conditions on the surface of the vinylester led to the scattering of the DIC data.

These results support the assumption of using two different values for the CTE in the solid and viscous (rubbery) state of the polymers. The initial state of the unreacted mix of hardener and resin is not the same as the viscous state of a cured polymer at higher temperature. However, the magnitude of different CTE between solid (s) and viscous (v) state for both polymers can be estimated.

\subsection{Modelling of shrinkage}

The model for the shrinkage of the materials contains both effects, the chemical shrinkage due to the formation of bonds forming denser networks and the thermal expansion because of the exothermal curing reaction of resin and hardener followed by a cooling phase. Thermal shrinkage takes place when the reaction rate decreases and the temperature drops.

In this model chemical shrinkage $(\Delta \varepsilon)$ only depends on time $(t)$, the maximal strain $\left(\varepsilon_{0}\right)$ and a reaction constant for the chemical reaction ( $\tau$ ). Shrinkage due to the chemical reaction starts at $t=t_{0}$ :

$\Delta \varepsilon=\varepsilon_{0} \cdot\left(e^{-\left(\frac{t-t_{0}}{\tau}\right)}-1\right) \cdot \Theta\left(t-t_{0}\right)$

The above equation describes the strain of the adhesives due to polymerisation (Fig. 8a). At $t=t_{0}$ the reaction starts and the material begins to shrink. In the following range the shrinkage is proportional to 
(a) Thermal expansion of the epoxy

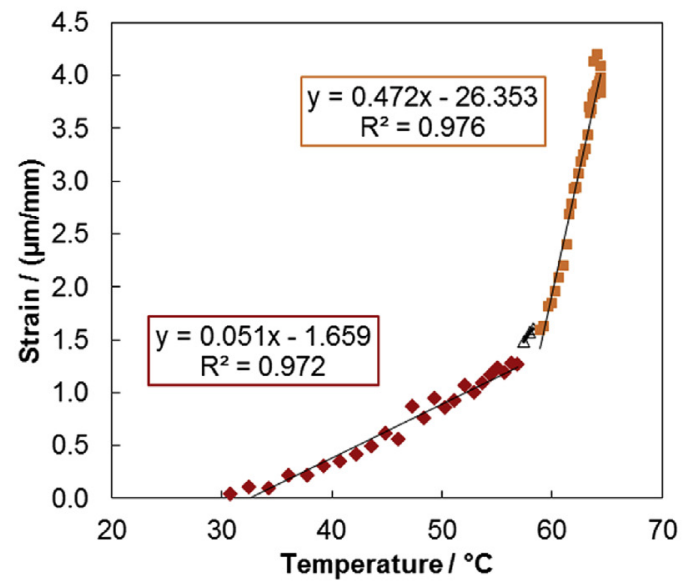

(b) Thermal expansion of the vinylester

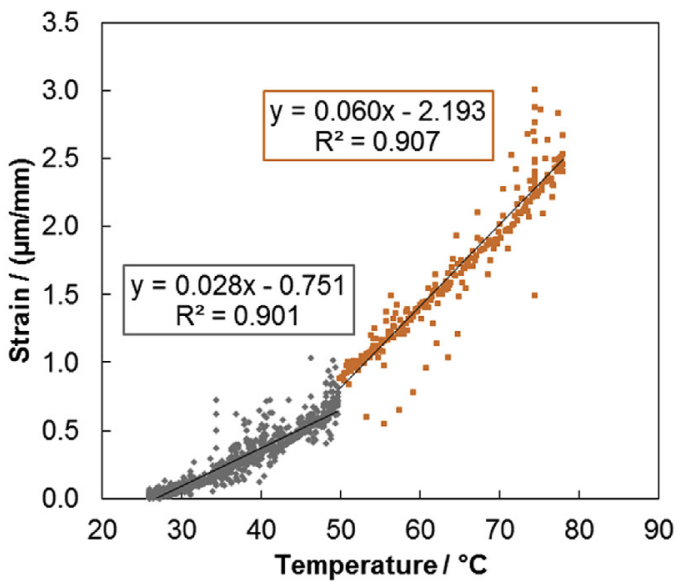

Fig. 7. Thermal expansion of epoxy (a) and vinylester (b) in two different ranges. The first slope represents the CTE in the solid state and the second slope at higher temperatures indicates the CTE in the viscous state above the $T_{g}$.

(a) Chemical shrinkage

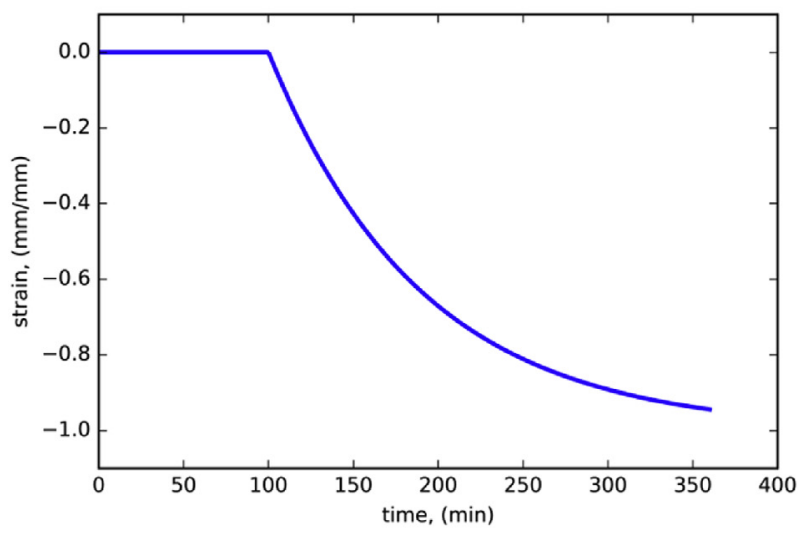

(b) Thermal expansion

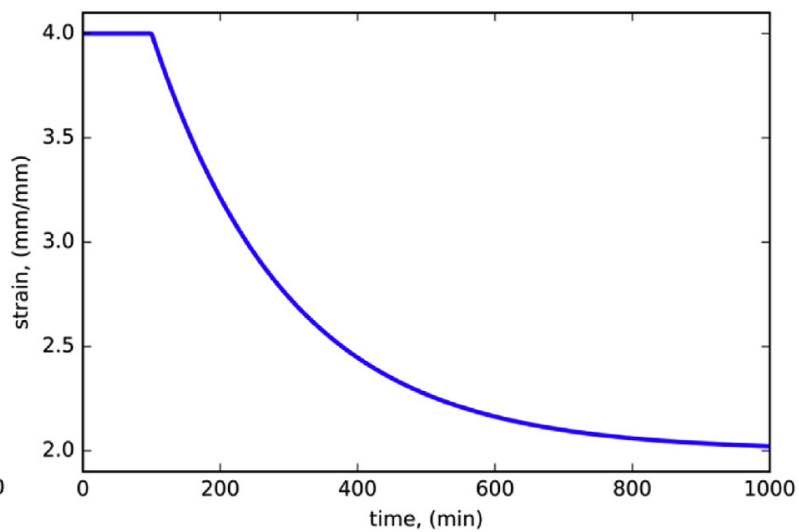

Fig. 8. The two parts of the shinkage model cover the chemical shrinkage reaction (a) and thermal expansion (b).

Table 1

Coefficient of thermal expansion (CTE) measured by DIC for epoxy and vinylester in solid (s) and viscous state (v).

\begin{tabular}{llll}
\hline Material & CTE $(\mathrm{v})$ & CTE $(\mathrm{s})$ & mean CTE $(\mathrm{v}+\mathrm{s})$ \\
\cline { 2 - 4 } & $\mathrm{mm} / \mathrm{mK}$ & $\mathrm{mm} / \mathrm{mK}$ & $\mathrm{mm} / \mathrm{mK}$ \\
\hline Epoxy & 0.472 & 0.051 & 0.261 \\
Vinylester & 0.060 & 0.028 & 0.044 \\
\hline
\end{tabular}

the not-yet-reacted polymer and therefore it follows an exponential decay. The Heaviside step function $(\Theta)$ equals 0 for negative arguments and 1 for positive arguments. In general many chemical reaction can be described by exponential equations depending on time and a reaction constant so it can be assumed that proper results can be obtained also for the chemical shrinkage of a polymer.

Due to the exothermal polymerisation reaction the temperature increases and leads to thermal expansion, competing with the curing related shrinkage. The thermal expansion or shrinkage depend on time,

Table 2

Results of the DIC shrinkage measurement and obtained CTE and constants from modelling of the measured shrinkage curve.

\begin{tabular}{|c|c|c|c|c|c|c|c|}
\hline \multirow[t]{2}{*}{ Material } & \multirow[t]{2}{*}{ Geometry } & \multirow{2}{*}{$\frac{\text { DIC strain, } \varepsilon}{\mathrm{mm} / \mathrm{m}}$} & \multirow{2}{*}{$\begin{array}{l}\text { Model strain, } \varepsilon \\
\mathrm{mm} / \mathrm{m}\end{array}$} & \multirow{2}{*}{$\frac{\operatorname{CTE}(1), \alpha_{l}}{\mathrm{~mm} / \mathrm{mK}}$} & \multirow{2}{*}{$\frac{\operatorname{CTE}(\mathrm{s}), \alpha_{s}}{\mathrm{~mm} / \mathrm{mK}}$} & \multirow{2}{*}{$\begin{array}{l}\text { Reaction constant, } \tau \\
\text { min }\end{array}$} & \multirow{2}{*}{$\frac{\text { Reaction delay, } \boldsymbol{t}_{0}}{\min }$} \\
\hline & & & & & & & \\
\hline Epoxy & round & -0.56 & -0.60 & 0.182 & 1.10 & 63.2 & 87.3 \\
\hline \multirow[t]{3}{*}{ Epoxy } & square & -0.91 & -0.88 & 0.202 & 0.443 & 41.0 & 129.6 \\
\hline & Mean: & -0.73 & -0.74 & 0.192 & 0.772 & 52.1 & 108.5 \\
\hline & Difference (\%): & 62.4 & 47.1 & 10.8 & 59.8 & 35.0 & 48.4 \\
\hline Vinylester & round & -10.49 & -10.31 & 0.142 & 0.062 & 0.30 & 2.30 \\
\hline \multirow[t]{3}{*}{ Vinylester } & square & -11.66 & -11.67 & 0.024 & 0.063 & 0.32 & 3.28 \\
\hline & Mean: & -11.08 & -10.99 & 0.083 & 0.062 & 0.31 & 2.79 \\
\hline & Difference (\%): & 11.1 & 13.2 & 83.1 & 2.8 & 6.8 & 42.7 \\
\hline
\end{tabular}


(a) EP, round

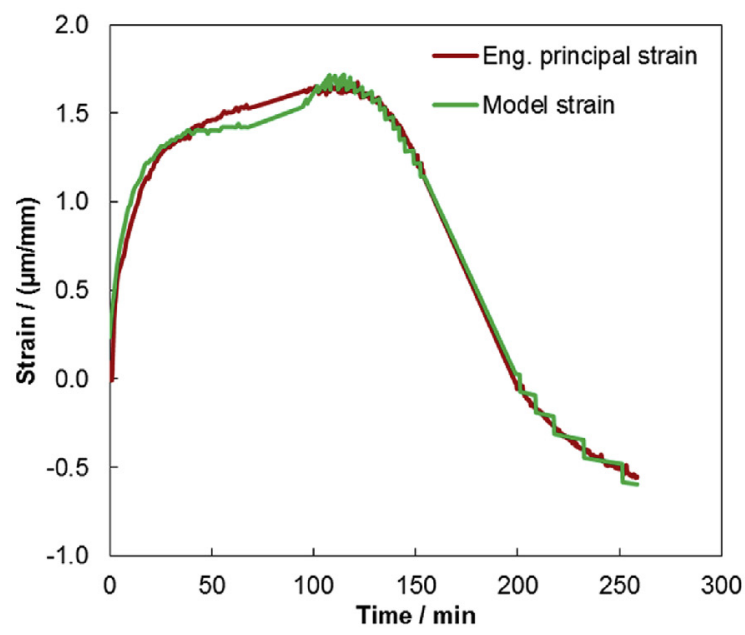

(c) EP, square

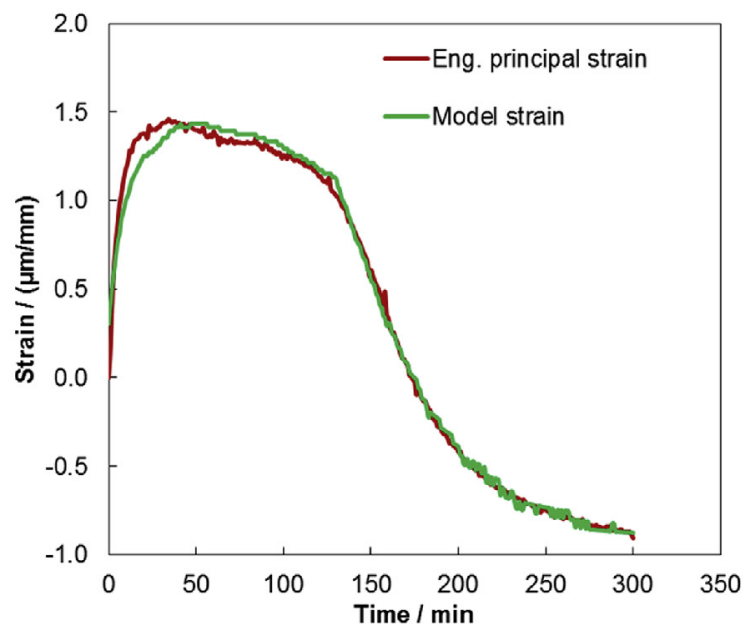

(b) VE, round

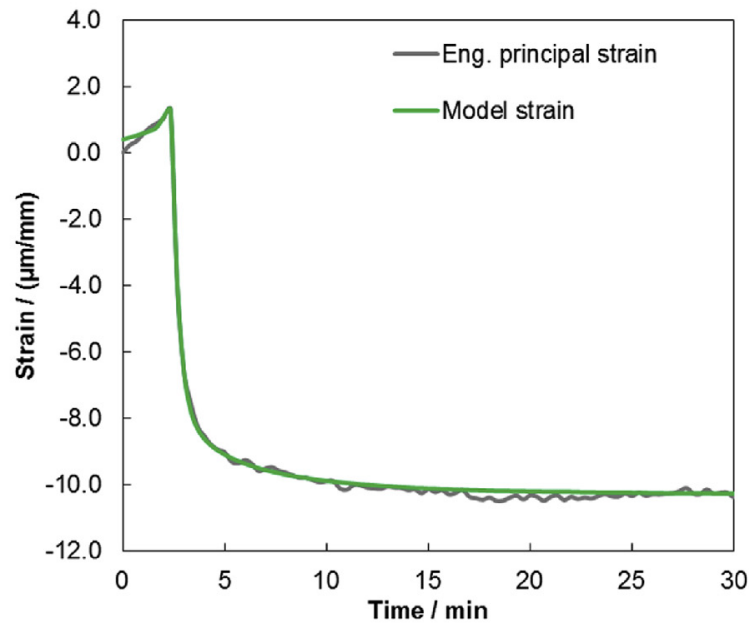

(d) VE, square

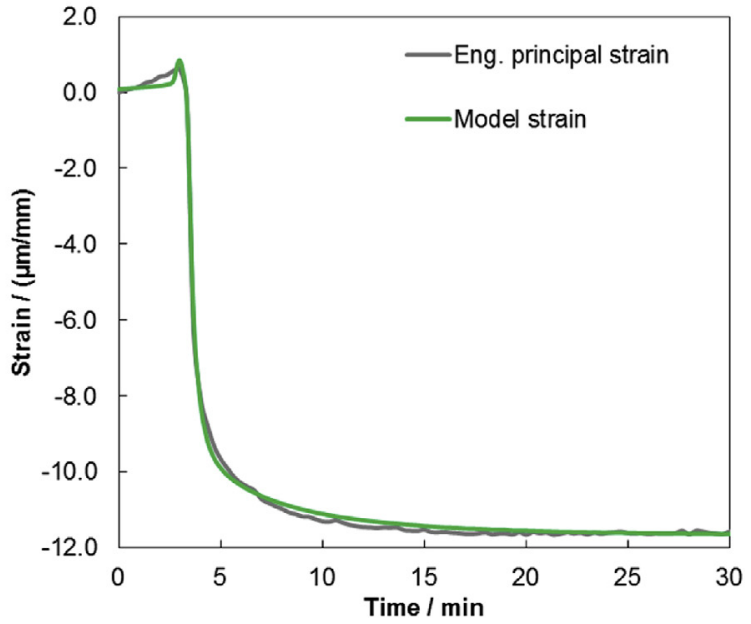

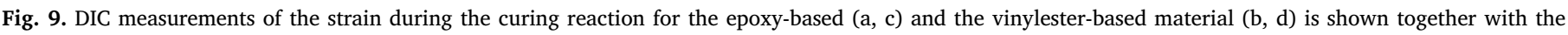
modelled strain for round and square sample geometry.

temperature difference $(\Delta T)$, the reaction constant and the two independent thermal expansion coefficients in solid $\left(\alpha_{s}\right)$ and liquid or notyet reacted polymer $\left(\alpha_{l}\right)$. The model consists of three parts: The first term describes the thermal expansion of the liquid resin before the main reaction starts, $t<t_{0}$. The second term starts at $t>t_{0}$ and describes the thermal expansion of the solid (already hardened resin) and the liquid phase (not-yet hardened resin). With increasing time, the amount of liquid phase diminishes as the amount of the solid phase increases. The shrinkage is described by the following equation:

$\Delta \varepsilon=\Delta T\left(\cdot \alpha_{l} \Theta\left(t_{0}-t\right)+\left(\left(\alpha_{l}-\alpha_{s}\right) \cdot e^{-\left(\frac{t-t_{0}}{\tau}\right)}+\alpha_{s}\right) \cdot \Theta\left(t-t_{0}\right)\right)$

The above equation is shown in Fig. 8b. Note that expansion corresponds to positive strain in the diagram.

Due to the obvious difference in thermal expansion coefficient of liquid and solid state of a polymer, the equation for thermal shrinkage/ expansion was split into two terms. For each domain the aforementioned model parameters $\left(\alpha_{l}, \alpha_{s}, \tau, t_{0}\right)$ were identified by fitting the measured strain vectors with model predictions obtained using the measured temperature differences as input. The resulting values are listed in Table 2. Comparing the modelled strain with DIC measurements demonstrates good agreement for both materials and both sample geometries (Fig. 9). The observed oscillations in the model predictions stem from the measurement noise in the temperature input data. For the epoxy in Fig. 9a,c the initial $\sim 100 \mathrm{~min}$ of the model slightly deviate from the experiment but show accordance after that time period. In case of the vinylester only small differences were obtained between model and experiment (Fig. 9b,d).

The CTE that were obtained from the shrinkage model (Table 2) can be compared with the values from the measurements of thermal expansion (Table 1). It can be seen, that the CTEs for the solid phase of the model (Table 2) are close to the CTEs of the viscous state from thermal expansion experiments. For the vinylester, the averaged calculated CTE (round and square samples) of the solid state $\alpha_{s}=0.062 \mathrm{~mm} / \mathrm{mK}$ is pretty much the same as that measured with the thermal expansion experiment $\alpha_{s}=0.060 \mathrm{~mm} / \mathrm{mK}$, and in the case of epoxy the averaged value from round and square specimen is $\alpha_{s}=0.772 \mathrm{~mm} / \mathrm{mK}$ and the viscous CTE $\alpha_{s}=0.472 \mathrm{~mm} / \mathrm{mK}$ are still close together. It might be concluded, that the CTE (s) from curing experiments describes a status nascendi of the material and therefore corresponds more to the CTE (v) from the thermal expansion experiment above the glass transition point.

From the thermal expansion experiments two different states of the cured polymers were obtained. First, the CTE of the solid (polymerized) state was calculated from the slope in the temperature-strain diagram. 
Secondly, the viscous state was reached at elevated temperature, which is connected to the glass transition region. This state is characterized by a drop of mechanical properties and change in other physical properties due to a certain softening of the material. In contrast to thermoplastics, which have a reversible liquid state, crosslinked thermosets have no liquid state at higher temperature.

For the shrinkage measurements it was assumed that no adhesion effects between material and the mold occurred. However, small influences due to adhesion cannot be fully excluded, since both materials are very sticky. For the DIC based evaluation of shrinkage, areas on the specimen surface were defined without considering the outer edges. Further, the model assumes homogenous shrinkage in the whole material, which can be confirmed at least for the surface by DIC measurements.

\section{Conclusions}

A contactless measurement of curing shrinkage and thermal expansion based on 3-dimensional optical digital image correlation (DIC) was presented. The method was tested on two different polymers (epoxy, vinylester) and provided excellent results in spite of the initially liquid state that complicated the application of stable yet good quality speckle patterns. The resulting curve was modeled considering chemical shrinkage due to the formation of a dense network and thermal expansion by the exothermal reaction of thermosets. The exothermal reaction enthalpy was measured by DSC and the temperature profile during the curing process was recorded with a thermocouple. Total shrinkage of the vinylester was approximately one order of magnitude higher compared to the epoxy. Thermal expansion measurement results and the shrinkage model showed good agreement considering that the coefficients of thermal expansion (CTE) for the viscous state should be better compared to the CTE(s) derived from the shrinkage model. The CTE(s) from the shrinkage model are values derived from an excited state of the system in status nascendi, which has not yet reached a stable configuration at room temperature. Reckon the configuration of the system, the CTE from the vinylester are more or less identical for both kind of experiments and the mean values for the epoxy are only $63 \%$ higher in the thermal expansion experiments, which in absolute values is in the range of half the difference of the CTE(s) determined between round and square specimens.

In conclusion, a powerful application of DIC as an accurate, contactless and flexible measurement method of thermoset shrinkage was presented, supported by a model approach that allows the separation of the curing process into the two simultaneous phenomena of chemical shrinkage and thermal expansion.

\section{Data availability}

The underlying experimental data consists of large series of pictures for the digital image correlation that cannot be made available online. However, the data is available on request.

\section{Acknowledgements}

The financial support by the Austrian Federal Ministry for Digital and Economic Affairs and the National Foundation for Research, Technology and Development is gratefully acknowledged, as is the partial financial support by the Austrian Science Fund (FWF): P 31203N32.

\section{Appendix A. Supplementary data}

Supplementary data to this article can be found online at https:// doi.org/10.1016/j.polymertesting.2019.106035.

\section{References}

[1] G. Singer, H. Rennhofer, G. Sinn, M. Unterlass, J. Wendrinsky, U. Windberger, H. Lichtenegger, Processing of Carbon Nanotubes and Carbon Nanofibers towards High Performance Carbon Fiber Reinforced Polymers, (2017).

[2] G. Singer, G. Sinn, K. Schwendtner, H.C. Lichtenegger, R. Wan-Wendner, Timedependent changes of mechanical properties of polymer-based composite materials for adhesive anchor systems, Compos. Struct. 196 (2018) 155-162.

[3] O. Moussa, A. Vassilopoulos, J. Castro, T. Keller, Long-Term Development of Thermophysical and Mechanical Properties of Cold-Curing Structural Adhesives Due to Post-Curing, (2013).

[4] M. Ganglani, S.H. Carr, J.M. Torkelson, Influence of cure via network structure on mechanical properties of a free-radical polymerizing thermoset, Polymer 43 (2002) 2747-2760.

[5] C. Damian, E. Espuche, M. Escoubes, Influence of three ageing types (thermal oxidation, radiochemical and hydrolytic ageing) on the structure and gas transport properties of epoxy-amine networks, Polym. Degrad. Stab. 72 (2001) 447-458.

[6] J. Zhou, J.P. Lucas, Hygrothermal effects of epoxy resin. Part I: the nature of water in epoxy, Polymer 40 (1999) 5505-5512.

[7] Y. Nawab, X. Tardif, N. Boyard, V. Sobotka, P. Casari, F. Jacquemin, Determination and modelling of the cure shrinkage of epoxy vinylester resin and associated composites by considering thermal gradients, Compos. Sci. Technol. 73 (2012) $81-87$.

[8] M. Haider, P. Hubert, L. Lessard, Cure shrinkage characterization and modeling of a polyester resin containing low profile additives, Compos. Appl. Sci. Manuf. 38 (2007) 994-1009.

[9] O.G. Kravchenko, S.G. Kravchenko, R.B. Pipes, Chemical and Thermal Shrinkage in Thermosetting Prepreg, Compos. Appl. Sci. Manuf. 80 (2016) 72-81.

[10] Y. Nawab, F. Jaquemin, P. Casari, N. Boyard, V. Sobotka, Evolution of chemical and thermal curvatures in thermoset-laminated composite plates during the fabrication process, J. Compos. Mater. 47 (2013) 327-339.

[11] P.J. Schubel, N.A. Warrior, C.D. Rudd, Surface quality prediction of thermoset composite structures using geometric simulation tools, Plastics, Rubber Compos. 36 (2007) 428-437.

[12] D.A. Tilbrook, G.J. Pearson, M. Braden, P.V. Coveney, Prediction of polymerization shrinkage using molecular modeling, J. Polym. Sci. B Polym. Phys. 41 (2003) 528-548.

[13] J.T. Zhang, M. Zhang, S.X. Li, M.J. Pavier, D.J. Smith, Residual stresses created during curing of a polymer matrix composite using a viscoelastic model, Compos. Sci. Technol. 130 (2016) 20-27.

[14] D.A. T, G.J. P, M. B, P.V. C, Prediction of polymerization shrinkage using molecular modeling, J. Polym. Sci. B Polym. Phys. 41 (2003) 528-548.

[15] D.U. Shah, P.J. Schubel, Evaluation of cure shrinkage measurement techniques for thermosetting resins, Polym. Test. 29 (2010) 629-639.

[16] I.-B. Lee, B.-H. Cho, H.-H. Son, C.-M. Um, B.-S. Lim, The effect of consistency, specimen geometry and adhesion on the axial polymerization shrinkage measurement of light cured composites, Dent. Mater. 22 (2006) 1071-1079.

[17] W.D. Cook, M. Forrest, A.A. Goodwin, A simple method for the measurement of polymerization shrinkage in dental composites, Dent. Mater. 15 (1999) 447-449.

[18] S.V. Hoa, P. Ouellette, T.D. Ngo, Determination of shrinkage and modulus development of thermosetting resins, J. Compos. Mater. 43 (2009) 783-803.

[19] W. Exner, A. Kühn, A. Szewieczek, M. Opitz, T. Mahrholz, M. Sinapius, P. Wierach, Determination of volumetric shrinkage of thermally cured thermosets using videoimaging, Polym. Test. 49 (2016) 100-106.

[20] J.D. James, J.A. Spittle, S.G.R. Brown, R.W. Evans, A review of measurement techniques for the thermal expansion coefficient of metals and alloys at elevated temperatures, Meas. Sci. Technol. 12 (2001) R1.

[21] C.Y. Ho, R.E. Taylor, Thermal Expansion of Solids, ASM International, 1998.

[22] N. McCormick, J. Lord, Digital image correlation, Mater. Today 13 (2010) 52-54.

[23] O. Kravchenko, S. Kravchenko, A. Casares, B. Pipes, Digital Image Correlation Measurement of Resin Chemical and Thermal Shrinkage after Gelation, (2015).

[24] D. Shah, Evaluation of Cure Shrinkage Measurement Techniques for Thermosetting Resins, (2010).

[25] I. Hamerton, L. Rapra Technology, Recent Developments in Epoxy Resins, Rapra Technology Ltd., Shawbury, Shrewsbury, Shropshire, UK, 1996.

[26] W. Brostow, S.H. Goodman, J. Wahrmund, 8 - epoxies, in: H. Dodiuk, S.H. Goodman (Eds.), Handbook of Thermoset Plastics, third ed., William Andrew Publishing, Boston, 2014, pp. 191-252. 